\title{
A Palette of Efficient and Stable Far-Red and NIR Dye Lasers
}

\author{
Edurne Avellanal-Zaballa ${ }^{1}$, Leire Gartzia-Rivero ${ }^{1}$, Jorge Bañuelos ${ }^{1, *}(\mathbb{C}$, \\ Inmaculada García-Moreno ${ }^{2}$, Antonia R. Agarrabeitia ${ }^{3}$, Eduardo Peña-Cabrera 4 \\ and Maria Jose Ortiz ${ }^{3}$ \\ 1 Dpto. Química Física, Universidad del País Vasco (UPV/EHU), Aptdo. 644, 48080 Bilbao, Spain; \\ edurne.avellanal@ehu.es (E.A.-Z.); leire.gartzia@ehu.es (L.G.-R.) \\ 2 Dpto. Sistemas de Baja Dimensionalidad, Superficies y Materia Condensada, Instituto Química-Física \\ "Rocasolano", IQFR-CSIC, Serrano 119, 28006 Madrid, Spain; i.garcia-moreno@iqfr.csic.es \\ 3 Dpto. Química Orgánica, Universidad Complutense, Ciudad Universitaria s/n, 28006 Madrid, Spain; \\ agarrabe@quim.ucm.es (A.R.A.); mjortiz@quim.ucm.es (M.J.O.) \\ 4 Dpto. Química, Universidad de Guanajuato, Noria Alta S/N, Guanajuato 36050, Mexico; eduardop@ugto.mx \\ * Correspondence: jorge.banuelos@ehu.es
}

Received: 17 August 2020; Accepted: 4 September 2020; Published: 7 September 2020

check for updates

Featured Application: Organic lasers active in the red-near-infrared spectral regions endowed with efficient and long-lasting emission.

\begin{abstract}
The disposal of long-wavelength-emitting sources is of paramount relevance in technology and biophotonics due to the low interference with the surroundings that these kinds of far-red and near-infrared radiations hold. As a result of the continued efforts carried out during the last few years by our research group to design new boron-dipyrromethene (BODIPY) dyes with improved photonic performance, two approaches were tested to develop a new generation of organic dyes able to display efficient and long-lasting laser emission in both target spectral regions. On the one hand, the annulation of aromatic benzofuran at the dipyrrin backbone leads to conformationally restricted dyes yielding photostable and bright laser emission beyond $600 \mathrm{~nm}$ at the far-red spectral region. On the other hand, a more pronounced shift to longer wavelengths reaching $725 \mathrm{~nm}$ at the near-infrared region is feasible, while keeping a reasonably high laser efficiency and tolerance to prolonged and intense pumping, based on aza-BODIPYs bearing peripheral aryl rings. These two complementary strategies yield a library of laser-emitting compounds comprising the 600-725 nm spectral region. Moreover, their laser performance is better than the commercially available dye lasers active in this spectral window.
\end{abstract}

Keywords: lasers; organic dyes; red-NIR emission; fluorescence; photostability

\section{Introduction}

The design of stable and bright photoactive systems able to emit in the far-red (within 600-700 nm spectral window) and especially in the near-infrared (NIR, beyond $700 \mathrm{~nm}$ ) spectral regions is an actively sought task in photonics [1-6]. This kind of long-wavelength emission is an attractive radiation source for telecommunications, as well as for optics and electronics (diodes, filters, logic gates, and optoelectronic devices in general) [7-10], owing to its low background interference with the surroundings which allows the light to travel long distances. They are also appealing as photosensitizers for solar cells because thegap between the highest occupied molecular orbital (HOMO) and lowest unoccupied molecular orbital (LUMO) matches the valence and conduction band gaps of 
semiconductors [11,12]. It is noteworthy that, in the last few years, NIR luminophores were intensively and successfully applied in biotechnology and biomedicine [13-16]. This kind of NIR radiation allows a deep penetration into tissues (up to $2-5 \mathrm{~cm}$ within the biological window, $\lambda>650 \mathrm{~nm}$ ), avoids autofluorescence of the surrounding biomolecules, and has lower toxicity. These features are ideal to apply high-resolution fluorescence imaging (such as super-resolution microscopy) for non-invasive diagnosis, sensing, and therapeutic purposes. In this regard, dye chemistry is a suitable tool to develop NIR absorbers and emitters thanks to the chemical versatility afforded by the highly accessible organic synthetic routes [17-20]. In particular, cyanines [21,22], porphyrines [23], and pthalocyanines [24], all of them featuring highly conjugated $\pi$-systems, are the most tested organic fluorophores for this aim (Figure 1). However, these fluorophores feature some drawbacks for their optimal performance. They require tedious synthetic and purification procedures. They display low fluorescence efficiency, mainly in the cyanine-based dyes, owing to the non-radiative deactivation channels afforded by their conformationally flexible structures and push-pull character. Moreover, they usually show poor photostability owing to photobleaching processes [25], likely related to the low energy gap between the excited and ground state. Finally, the chemical versatility for postfunctionalization is limited in these molecular skeletons.
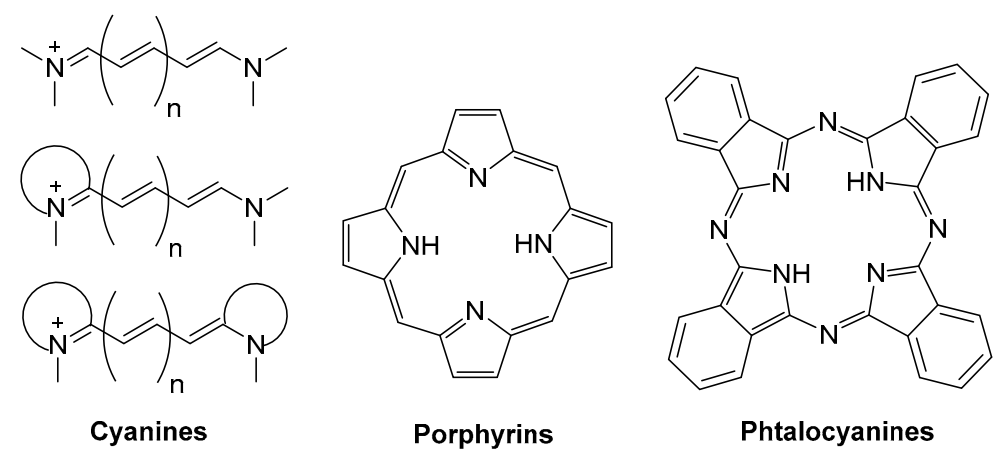

Figure 1. Molecular structures of the most commonly tested red and near-infrared (NIR) dyes based on cyanine backbones.

To overcome such limitations, the design of a new generation of organic dyes endowed with absorption and emission bands within the biological window, easily accessed and derivatized, and displaying bright and long-lasting emissions is a highly demanded issue. In this regard, the renowned boron-dipyrromethene (BODIPY) dyes, which can be considered as the little sister of porphyrins or cyclic cyanines, drew the attention of researchers. Their boron-dipyrrin core is robust and stable (both chemically and photochemically) [26], and their photophysical signatures can be modulated by the substitution pattern [27]. Furthermore, BODIPYs can be easily accessed through many synthetic routes and straightforwardly functionalized to deeply shift their spectral bands toward the red edge of the electromagnetic spectrum [28]. In the literature, several chemical modifications were reported to this aim [29]. The main strategies are the following:

(1) The induction of intramolecular charge transfer (ICT) phenomena in BODIPYs via the grafting of electron donor and acceptor moieties (push-pull dyes) [30,31]. The emission from the ICT is strongly shifted to longer wavelengths. However, it is usually very weak and very sensitive to the environmental conditions.

(2) The extension of the delocalized $\pi$-system through aromatic frameworks. To this aim, aromatic rings are annulated at the dipyrrin core, leading to conformationally restricted BODIPYs $[32,33]$ or directly connected to the core via single bonds [34,35]. Moreover, according to this last procedure, two (bis-BODIPYs) [36] or several BODIPY units (oligomers) [37] can be linked through spacers allowing electronic coupling or directly fused through aromatic rings (fused bis-BODIPYs) [38-40]. In this way, NIR absorbers are achieved. However, the electronic 
rearrangement upon resonant interactions between the electronic clouds of the BODIPYs usually leads to poorly emissive compounds.

(3) The replacement of the meso carbon by an aza group (aza-BODIPY) [41-43]. Such simple modification induces pronounced bathocromic shifts [44], which can be enlarged spanning the delocalize $\pi$-system, as in the preceding point. Indeed, the aza-BODIPY dye family stands out as a promising alternative to design brighter, more stable, and compact NIR emitters than the classic phtalocyanines.

Against this background, in the last few years, we intensively explored the rich chemistry of BODIPY dyes. As a result, we designed, synthesized, and characterized a library of BODIPY-based far-red and NIR emitting dyes, covering the spectral region ranging from 650 to $750 \mathrm{~nm}$, and applied them as lasers. The benefits of these kinds of coherent and powerful radiation holds are well known. As a matter of fact, many laser-based devices and technologies are applied intensively and successfully in industry, communications, surgery, and daily life. Among the different kinds of available lasers, those based on organic molecules as active media are in the spotlight $[45,46]$. The main advantage of dye lasers or organic lasers is the chemical versatility afforded by synthetic tools. Thus, there are a wide chart of commercially available laser dyes across the ultraviolet, visible, and NIR spectral regions. Moreover, they display a broad band enabling a certain sintonization range, not usually available in those lasers based on inorganic photoactive media, along with the generation of ultrashort pulses (in the femtosecond scale). This last property is appealing to study fast molecular dynamics in the excited state by advanced spectroscopic techniques. On the other hand, the main drawback of organic lasers is their limited photostability. To circumvent such restriction, complex and bulky experimental set-ups are required. Therefore, to offset this deficiency, we developed red and NIR dye lasers with improved photonic performance, particularly focused on enhancing the photostability, i.e., benzofuran-fused BODIPYs (Section 2) as laser dyes emitting in the far-red region of the visible spectral region [47] and polyarylated aza-BODIPYs (Section 3) for the NIR spectral region (Figure 2) [48]. Hereafter, we overview the photophysical and laser properties of these novel BODIPYs designed in our laboratories and compare their photonic performance with commercially available laser dyes working in the same spectral region.

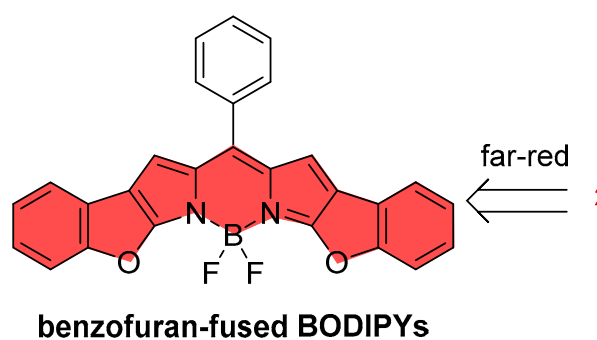

benzofuran-fused BODIPYs

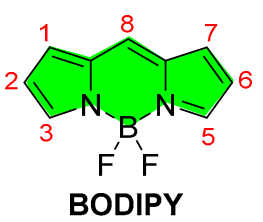

BODIPY

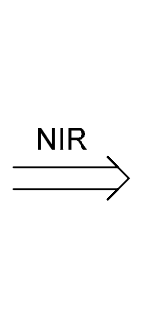

polyarylated aza-BODIPYs

Figure 2. Molecular structure of the renowned boron-dipyrromethene (BODIPY) chromophore and the chemical modification carried out to push the spectral bands toward the red edge of the visible spectrum and NIR region.

\section{Red-Emitting Benzofuran-Fused BODIPYs}

The laser dyes designed to cover the far-red region of the visible spectrum are based on a BODIPY core bearing fused benzofuran rings with different substitution patterns (fluorine, alkyls, linear alcoxys, or additional fused rings) [47]. The corresponding molecular structures and main photophysical and laser data are summarized in Figure 3.

The fusion of benzofuran rings at chromophoric positions 2 and 3 and their symmetrical positions 5 and 6 induces the expected bathochromic shift. In fact, the theoretically predicted molecular orbitals show that the $\pi$-system is extended through the whole molecule (Figure 4). The exception is the 8-aryl 
group, not taking part in the delocalized $\pi$-system owing to its twisted disposition [26]. The presence of electron donor moieties (RED7, RED9, and RED11) and mainly the further fusion of more aromatic groups (RED8) induce a more pronounced shift to the red edge, from 575 to $610 \mathrm{~nm}$ (Figures 3 and 4). Such spanning of the aromaticity is reflected in a remarkable enhancement of the absorption probability (molar absorption coefficient), being twice that recorded for simpler BODIPYs, and reaching values approaching 200,000 $\mathrm{M}^{-1} \cdot \mathrm{cm}^{-1}$ (Figures 3 and 4) [47]. The corresponding fluorescence bands are located in a spectral window ranging from 595 to $625 \mathrm{~nm}$. The spectral proximity between the absorption and fluorescence spectra (low Stokes shift) suggests a low geometrical rearrangement upon excitation (Figure 4). The cyclization of the substituents limits the conformational freedom, and these constrained geometries hinder non-radiative relaxation processes [49]. As a result, the recorded fluorescence efficiencies are high, surpassing $80 \%$ in most cases (regardless of the applied substitution pattern) and even approaching $100 \%$ in some cases (Figure 3). The exceptions to this behavior are due to an increase in the non-radiative deactivation processes induced by the substitution pattern. For instance, in RED5, the combination of electron donor benzofuran with electron formaldehyde at the para-8-aryl favors charge separation and reduces the emission capability [47]. In RED8 and RED11, the geometrical tensions induced by the additional fused phenyls could promote the bending of the chromophore, thereby distorting the planarity and increasing the internal conversion [47].

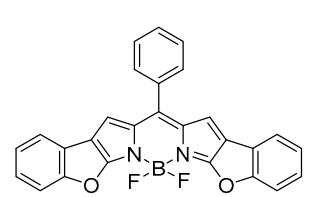

RED1

$\lambda_{\mathrm{ab}}=581-\varepsilon_{\max }=12.4$

$\lambda_{\mathrm{fl}}=596-\phi=0.82$

$\lambda_{\text {la }}=647-\% \mathrm{Eff}=44$

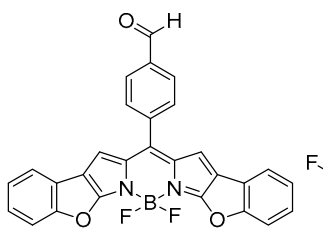

RED5

$\lambda_{\mathrm{ab}}=587-\varepsilon_{\max }=11.9$

$\lambda_{\mathrm{Fl}}=610-\phi=0.40$

$\lambda_{\text {la }}=636-\%$ Eff $=28$

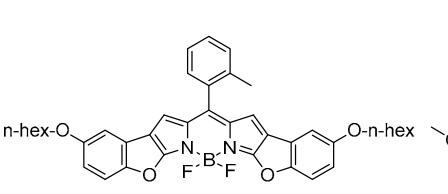

RED9

$\lambda_{\mathrm{ab}}=587-\varepsilon_{\max }=15.7$

$\lambda_{\mathrm{fl}}=602-\phi=0.93$

$\lambda_{\text {la }}=657-\%$ Eff $=43$

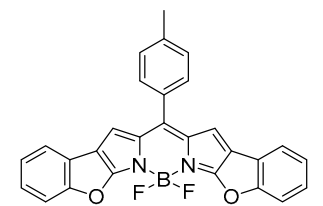

RED2

$\lambda_{\mathrm{ab}}=579-\varepsilon_{\max }=17.0$

$\lambda_{\mathrm{fl}}=594-\phi=0.92$

$\lambda_{\text {la }}=646-\% \mathrm{Eff}=44$

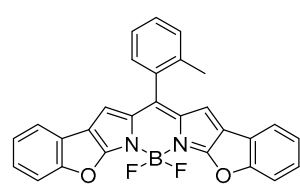

RED3

$\lambda_{\mathrm{ab}}=582-\varepsilon_{\text {max }}=16.9$

$\lambda_{\mathrm{fl}}=595-\phi=0.93$

$\lambda_{\mathrm{la}}=649-\% \mathrm{Eff}=48$

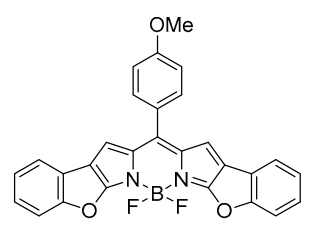

RED4

$\lambda_{\mathrm{ab}}=578-\varepsilon_{\max }=15.7$

$\lambda_{\mathrm{fl}}=594-\phi=0.83$

$\lambda_{\text {la }}=645-\%$ Eff $=45$

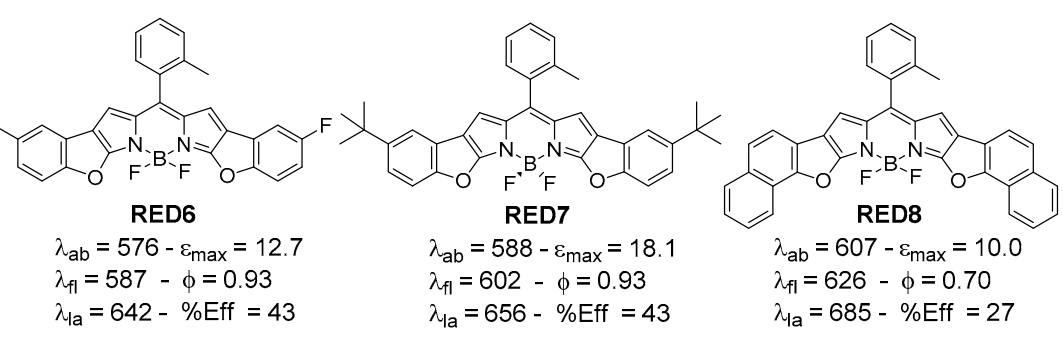

Figure 3. Molecular structures of the benzofuran-fused BODIPYs and their corresponding main photophysical (at diluted solutions of $2 \mu \mathrm{M}$ ) and laser (at the optimal dye concentration ranging from 0.5 to $1.2 \mathrm{mM})$ data in ethyl acetate: absorption wavelength $\left(\lambda_{\mathrm{ab}}\right.$, in $\left.\mathrm{nm}\right)$, molar absorption coefficient $\left(\varepsilon_{\max }\right.$, in $\left.10^{4} \mathrm{M}^{-1} \cdot \mathrm{cm}^{-1}\right)$, fluorescence wavelength $\left(\lambda_{\mathrm{fl}}\right.$, in $\left.\mathrm{nm}\right)$, quantum yield $(\phi)$, laser wavelength $\left(\lambda_{\mathrm{la}}\right.$, in $\mathrm{nm}$ ), and efficiency (\% Eff). The laser emission of RED11 could not be recorded due to lack of solubility at the required high concentration.

The excellent photophysical properties of these dyes led to an outstanding laser performance in the far-red region. Strong laser emissions were recorded spanning the spectral region from $615 \mathrm{~nm}$ to 
$660 \mathrm{~nm}$ (Figure 4) with efficiencies approaching 50\% (see RED1-4, RED6-7, and RED9-10 in Figure 3). The lasing properties followed the photophysical trends. The bathochromic shift of the laser wavelength correlated with a shift of the fluorescence band with the substitution pattern (see RED8 in Figure 3). Moreover, those dyes showing the lowest fluorescence efficiency displayed the poorest laser efficiency (see RED5 and RED8 in Figure 3). It is noteworthy that those dyes with flat molecular geometries are prone to stacking with each other, leading to the formation of aggregates [50]. This phenomenon is active at the high optical densities required to record the laser action. Indeed, we detected dual laser emissions. Thus, at high pump energies, a long wavelength emission is registered flanking that recorded at lower pump energies. The latter emission is the expected one, but the former emission is assigned to the presence of aggregates (photoinduced excimers or superexciplexes). These molecular entities are only detected under strong pumping, but not under standard excitation as in photophysical measurements [51]. These aggregates are able to lase and increase the sintonization range of the laser emission.

Photostability is a key parameter ruling the operative lifetime of dyes and light-driven devices [25]. This signature was estimated as the amount of energy required to reduce $10 \%$ of the laser-induced fluorescence (that is, to retain $90 \%$ of the initial emission; see inset of Figure 4). All the dyes showed a reasonable tolerance to a continuous and strong irradiation regime. In some cases, the photostability was exceptional (reaching $7 \mathrm{GJ} / \mathrm{mol}$ in RED6, likely owing to the stability provided by the strongly bounded fluorine atoms [52]), yielding extremely long-lasting emission. It is noteworthy that the lowest photostabilities correspond to the dyes bearing the 8-phenyl para-substituted (Figure 4), with a metoxy group in RED4 and a formaldehyde moiety in RED5. On the other hand, the highest photostabilities were attained in dyes where the benzofuran moiety was functionalized (with fluorine, tert-butyl or aliphatic alcoxy chains, RED6-7 and RED9, respectively).

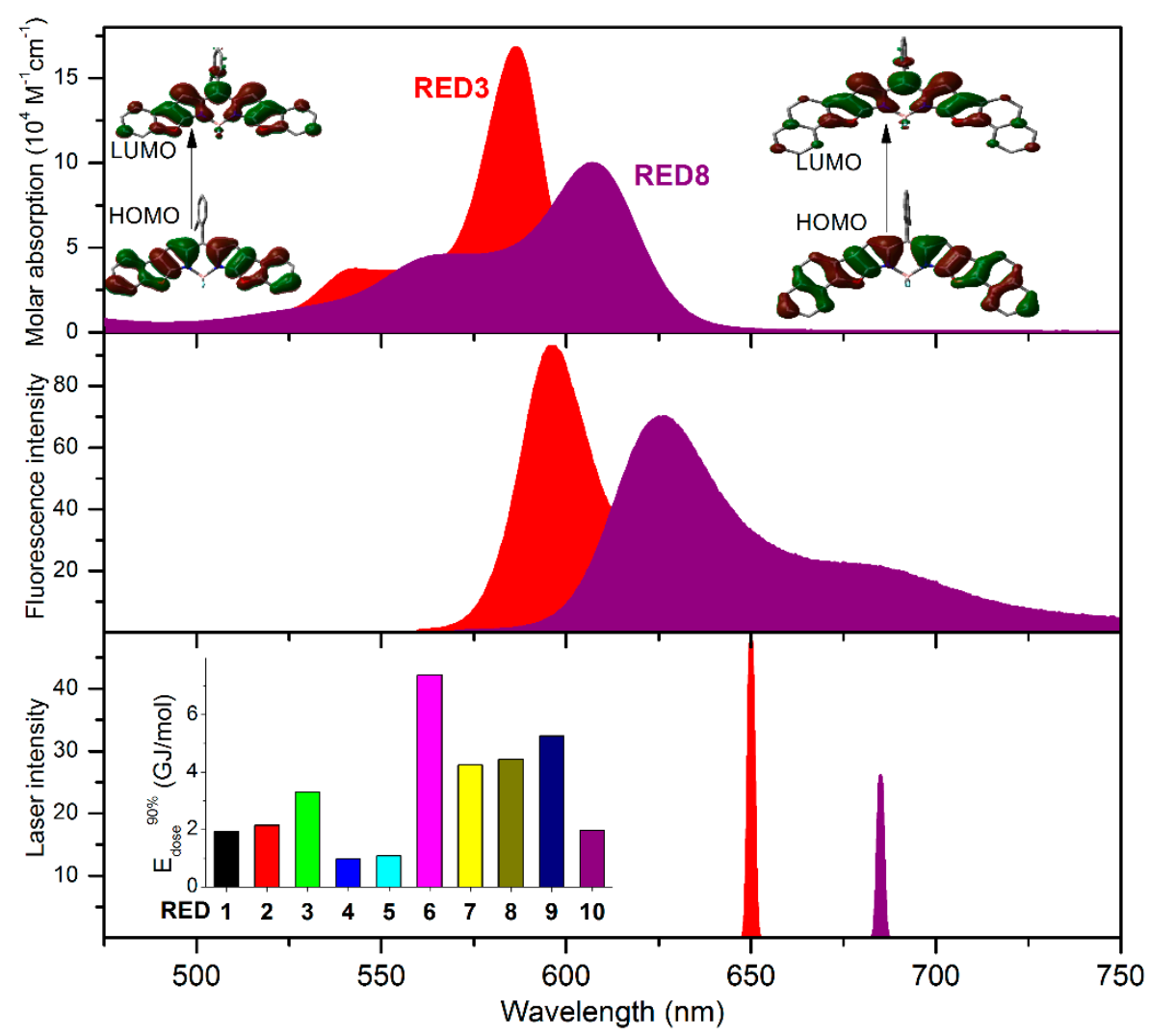

Figure 4. Absorption, fluorescence and laser spectra of representative benzofuran-fused BODIPYs (RED3 and the more $\pi$-extended RED8) in ethyl acetate. Inset: top, contour maps (HOMO and LUMO) of the frontier orbitals involved in the electronic transition; bottom, photostability calculated as the energy dose required to decrease $10 \%$ of the laser-induced fluorescence $\left(\mathrm{E}_{\text {dose }}{ }^{90 \%}\right)$. 
To highlight the notable photonic performance of these laser dyes, we compared the laser behavior of the benzofuran-fused BODIPYs with a commercially available BODIPY working in the same spectral region (PM650, where the number accounts for the laser emission wavelength) [53]. The photostability of PM650 (6.3 GJ/mol) is of the same order (up to 7.4 in RED6) owing to the robustness of the BODIPY chromophore, but the efficiency is clearly enhanced (from 31\% in PM650 to 48\% in RED3). Therefore, we were able to improve the light-harvesting ability and retain the long-lasting emission efficiency typical of the BODIPYs while pushing it into the far-red edge of the visible spectrum.

\section{NIR-Emitting Polyarylated Aza-BODIPYs}

To induce a more pronounced shift of the laser emission to longer wavelengths, we focused on aza-BODIPYs bearing a $\pi$-extended delocalize framework through peripheral aromatic substitutions at the dipyrrin core (Table 1) [48].

Table 1. Molecular structure of the NIR aza-BODIPYs and their corresponding main photophysical (at diluted solutions of $2 \mu \mathrm{M}$ ) and laser (at the optimal dye concentration ranging from $0.25 \mathrm{mM}$ to $1 \mathrm{mM}$ ) data in ethyl acetate: absorption wavelength $\left(\lambda_{\mathrm{ab}}\right)$, molar absorption coefficient $\left(\varepsilon_{\max }\right)$, fluorescence wavelength $\left(\lambda_{\mathrm{fl}}\right)$, quantum yield $(\phi)$, laser wavelength $\left(\lambda_{\mathrm{la}}\right)$, and efficiency (\% Eff). The corresponding O-aza-BODIPY in NIR1 could not be isolated owing to its chemical instability.

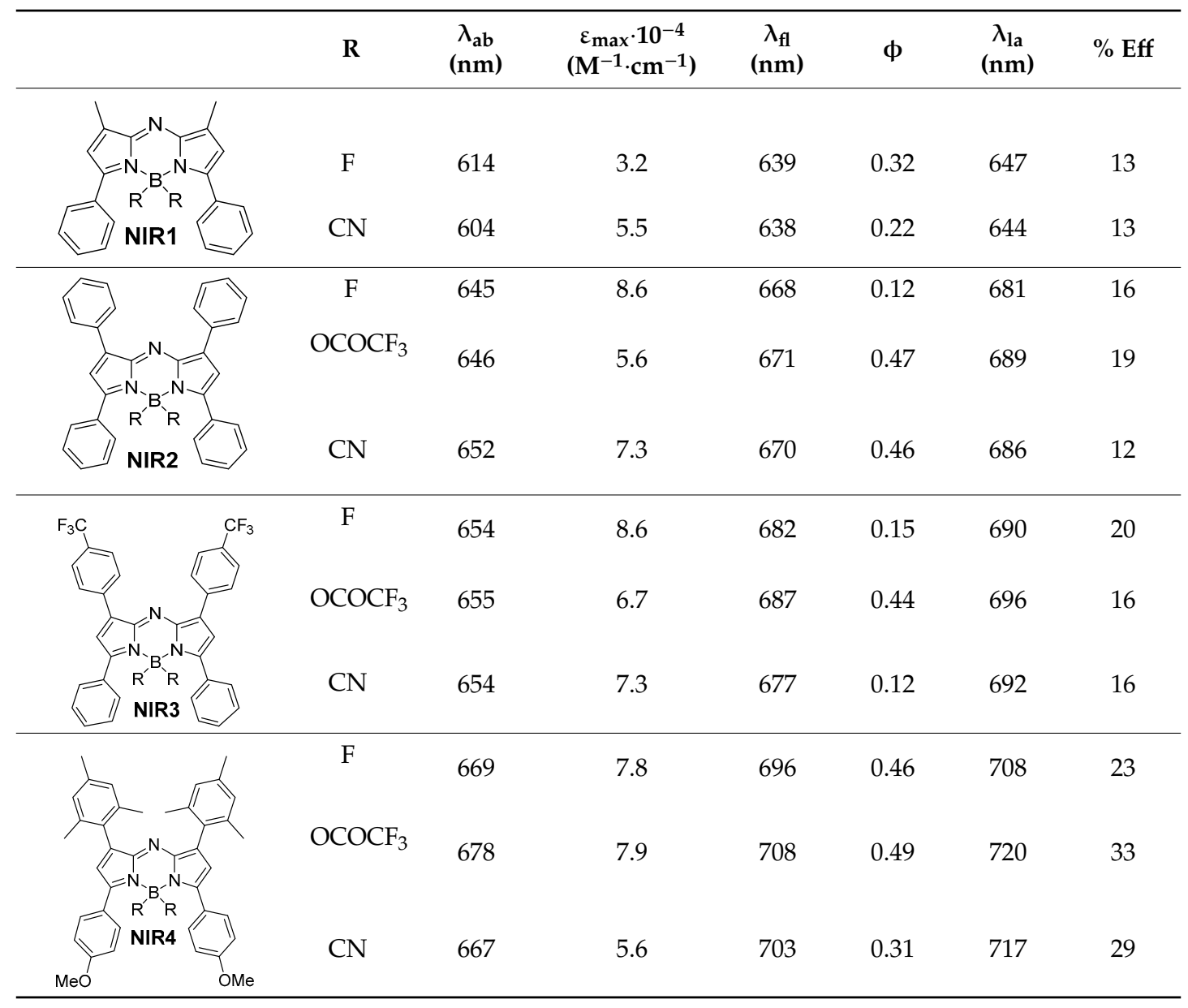

The replacement of the carbon at position 8 by an aza group is a simple but effective way to induce the desired bathochromic shift without enlarging the molecular size. The electronegativity of the nitrogen at such a chromophoric position induces a preferred stabilization of the LUMO state, reducing the energy gap [44]. Such a red shift can be reinforced through peripheral substitutions with aryl groups and electron-rich moieties to extend the $\pi$-system and reach emission in the NIR region. 
In fact, in F-aza-BODIPYs, the spectral bands, both absorption and fluorescence, shift progressively to longer wavelengths (Table 1 and Figure 5) as the number of appended unrestricted phenyls increases (from NIR1, with two peripheral phenyls, to NIR2 with four rings), and even further upon functionalization of the peripheral aryls with electron donor groups (like methoxy in NIR4).

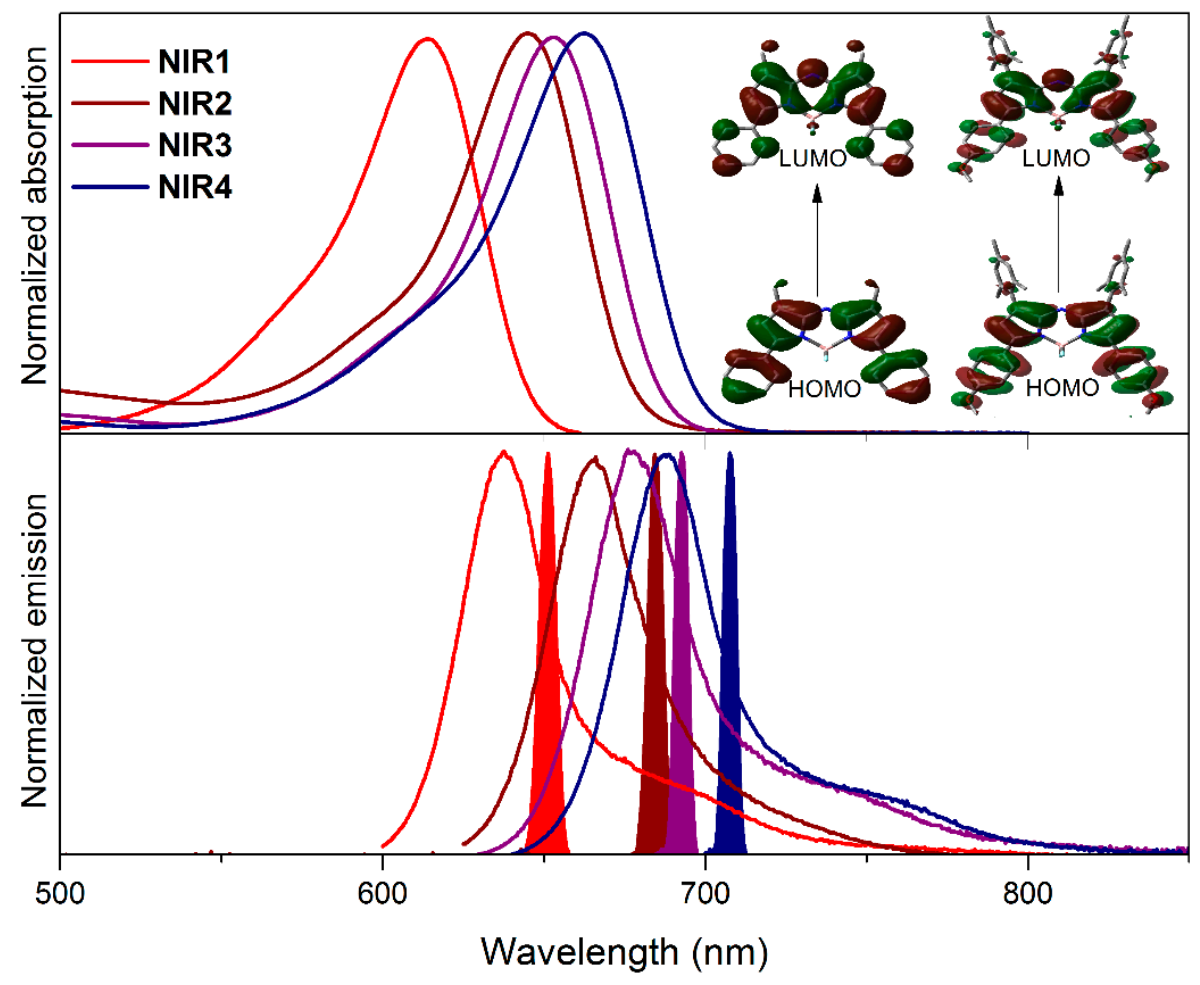

Figure 5. Absorption, fluorescence, and laser (filled) spectra of two representative F-aza-BODIPYs (NIR1 and NIR4) in ethyl acetate. Inset: corresponding contour maps (HOMO and LUMO) of the frontier orbitals involved in the electronic transition.

The fluorescence response of the F-aza-BODIPYs was lower (around 45\%, Table 1) than that recorded for the conformationally restricted BODIPYs (see section with values up to $95 \%$ in Figure 3). This was expected in terms of the energy gap law [54]. A lowering of the energy gap enhances the vibrational coupling of the involved electronic states and, hence, the internal conversion probability. Nevertheless, fluorescence efficiencies of around $45 \%$ are notable in the NIR region. Such emission depends mainly on the conformational freedom of the aryls at positions 1 and 7 of the chromophoric core. Thus, the fluorescence of NIR2 and NIR3 decreased to $12 \%$, whereas the locking of the free motion of such 1,7-rings (sterically hindered mesityl group instead of phenyl) implied a recovery of the fluorescence signal (up to $46 \%$ in NIR4, Table 1). In this regard, NIR4 is the most appealing dye among the tested ones owing to its high fluorescence efficiency at longer wavelength [48].

All these dyes showed laser bands tunable from 650 to $700 \mathrm{~nm}$, in good correlation with the evolution of the fluorescence band position depending on molecular structure (Figure 5). The laser efficiencies ranged from $13 \%$ to $23 \%$, with NIR4 again being the dye with the best laser performance in agreement with the photophysical signatures. Nevertheless, the most outstanding result was the excellent photostability shown by these dyes (up to $44 \mathrm{GJ} / \mathrm{mol}$, Figure 6). These F-aza-BODIPYs showed a noticeable four-fold enhancement with respect to commercial laser dyes emitting in the same spectral region, such as the oxazine dye called Nile Blue [55], while retaining similar laser efficiencies (around $30 \%$ in Nile Blue). In other words, these F-aza-BODIPYs display efficient and long-lasting laser signals, drastically improving the photostability of commercially available NIR laser dyes. 


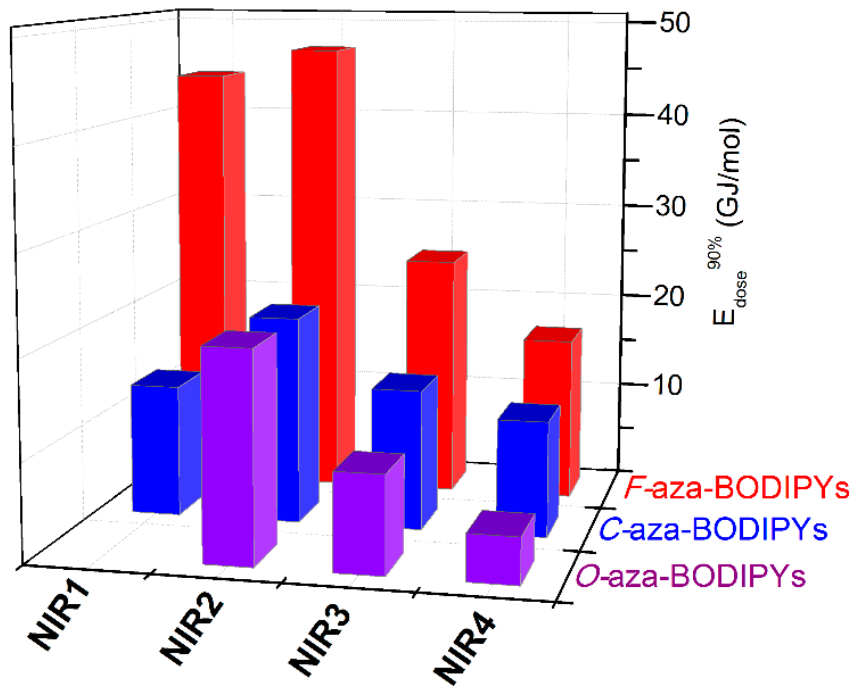

Figure 6. Photostability (energy dose required to decrease $10 \%$ of the laser-induced fluorescence $\mathrm{E}_{\mathrm{dose}}{ }^{90 \%}$ ) of the $\mathrm{F}_{-}, \mathrm{C}$-, and $\mathrm{O}$-aza-BODIPYs in ethyl acetate.

Trying to ameliorate both laser parameters, efficiency and photostability, we carried out structural modifications at the boron atom rendering the corresponding $C$ - and $O$-aza-BODIPYs (Table 1 ). As a common rule in BODIPYs, the replacement of fluorine atoms (F-BODIPYs) by alcoxys (O-BODIPYs) or alkyls (C-BODIPYs) improved the photonic response [56-58]. Thus, we developed the corresponding $\mathrm{O}$ - and $\mathrm{C}$-aza-BODIPYs, replacing the fluorine atoms at the boron center with electron acceptor trifluoroacetoxy and cyane moieties [48], respectively (Table 1). According to previous results in simpler BODIPYs, both substituents were the most suitable to improve the photonic performance [56,57]. Such chemical modification has a minor impact on the spectral band positions (overall a slight bathochromic shift) since the boron atom does not take part in the dipyrrin aromatic framework [48]. However, such substitution has a marked impact on the fluorescence efficiency, and it depends on the peripheral substitution pattern of the dipyrrin (Table 1). Thus, in the $O$-aza-BODIPYs derived from NIR2 and NIR3, a clear enhancement (four-fold) of the fluorescence response was recorded. The fluorescence response of the $O$-aza-BODIPY derived from NIR4 remained almost the same as that of the corresponding F-aza-BODIPYs. Moreover, the $C$-aza-BODIPYs did not improve the fluorescence response shown by the $O$-aza-BODIPYs, and NIR2 was better than the corresponding $F$-aza-BODIPY. Conversely, such improved fluorescence was not reflected in a marked increase of the lasing efficiency. Improved laser efficiencies were only recorded in the derivatives of NIR2 and NIR4. Additionally, the photostability of the $O$ - and $C$-aza-BODIPYs clearly dropped (two- or three-fold depending of the dye, Figure 6) with regard to the parent $F$-aza-BODIPYs. Moreover, we observed that the $C$-aza-BODIPYs and especially the $O$-aza-BODIPYs were chemically unstable, and the solution lost its color with aging time. Likely, these two factors, low chemical and photochemical stability, could explain the existing mismatch between the fluorescence and laser efficiencies. Such low stability was strengthened under strong pumping in the laser resonator cavity.

\section{Conclusions}

The rich and versatile chemistry of BODIPYs is a suitable tool to design a chart of photoactive dyes as efficient and stable tunable lasers within the spectral window ranging from 600 to $725 \mathrm{~nm}$. Within the far-red region of the visible spectrum, conformationally restricted benzofuran-fused BODIPYs are a suitable option. Their flat and rigid aromatic framework allows a huge absorption of the pumping light and avoids non-radiative deactivation funnels. Therefore, these dyes yield efficient (approaching the $50 \%$ ) and stable laser signals ranging from 600 to $650 \mathrm{~nm}$. To further push the laser emission toward the NIR region, polyarylated aza-BODIPYs are more suitable. Strikingly, the chemical modification at 
the boron atom is not a recommended strategy because the chemical and photochemical stability of the dye is reduced. Therefore, $F$-aza-BODIPYs are the best design to record notable laser emission from 650 to $725 \mathrm{~nm}$, which is outstanding, mainly due to their high photostabilities.

Both set of dyes surpass the laser performance of commercially available laser dyes working in the same spectral region. On the one hand, the benzofuran-fused BODIPYs markedly improve the brightness of the laser emission in the far-red region. On the other hand, the aza-BODIPYs greatly increase the photostability, leading to long-lasting tunable laser emissions in the NIR region.

Furthermore, these molecular scaffolds might be envisaged as promising fluorescent probes for bioimaging purposes owing to their efficient light absorption within the biological window and ulterior display of bright fluorescent images over long exposure periods.

Author Contributions: Synthesis of benzofuran-fused BODIPYs, E.P.-C.; synthesis of aza-BODIPYs, A.R.A. and M.J.O.; photophysical and laser measurements, E.A.-Z., supervision of laser experiments, I.G.-M.; supervision of photophysical experiments, writing—original draft preparation, and editing, J.B.; writing-review, L.G.-R. All authors have read and agreed to the published version of the manuscript.

Funding: This research was funded by the Spanish Ministerio de Economia y Competitividad (MINECO), grant number MAT2017-83856-C3-1-P, 2-P and 3-P, and Gobierno Vasco, grant number IT912-16.

Acknowledgments: E.A.Z. thanks Gobierno Vasco for a predoctoral fellowship.

Conflicts of Interest: The authors declare no conflict of interest.

\section{References}

1. Zampetti, A.; Minotto, A.; Cacialli, F. Near-Infrared (NIR) Organic Light-Emitting Diodes (OLEDs): Challenges and Opportunities. Adv. Funct. Mater. 2019, 29, 1807623. [CrossRef]

2. Zhao, Q.; Sun, J.Z. Red and near infrared emission materials with AIE characteristics. J. Mater. Chem. C 2016, 4, 10588-10609. [CrossRef]

3. Qu, Z.; Shen, J.; Li, Q.; Xu, F.; Wang, F.; Zhang, X.; Fan, C. Near-IR emissive rare-earth nanoparticles for guided surgery. Theranostics 2020, 10, 2631-2644. [CrossRef] [PubMed]

4. Li, J.-B.; Liu, H.-W.; Fu, T.; Wang, R.; Zhang, X.-B.; Tan, W. Recent Progress in Small-Molecule Near-IR Probes for Bioimaging. Trends Chem. 2019, 1, 224-234. [CrossRef] [PubMed]

5. Wunsch, A.; Matuschka, K. A Controlled Trial to Determine the Efficacy of Red and Near-Infrared Light Treatment in Patient Satisfaction, Reduction of Dine Lines, Wrinkles, Skin Roughness, and Intradermal Collagen Density Increase. Photomed. Laser Surg. 2014, 32, 93-100. [CrossRef] [PubMed]

6. Pansare, V.; Hejazi, S.; Faenza, W.; Prud'Home, R.K. Review of Long-Wavelength Optical and NIR Imaging Materials: Contrast Agents, Fluorophores and Multifunctional Nano Carriers. Chem. Mater. 2012, 24, 812-827. [CrossRef] [PubMed]

7. Bünzli, J.-C.G.; Eliseeva, S.V. Lanthanide NIR luminescence for telecommunications, bioanalyses and solar energy conversion. J. Rare Earths 2010, 28, 824-842. [CrossRef]

8. Kaur, H.; Sundriyal, S.; Oachauri, V.; Ingebrandt, S.; Kim, K.-H.; Sharma, A.L.; Deep, A. Luminescent metal-organic frameworks and their composites: Potential future materials for organic light emitting displays. Coord. Chem. Rev. 2019, 41, 213077. [CrossRef]

9. Dou, L.; Liu, Y.; Hong, Z.; Li, G.; Yang, Y. Low-Bandgap Near-IR Conjugated Polymers/Molecules for Organic Electronics. Chem. Rev. 2015, 115, 12633-12665. [CrossRef]

10. Kim, K.H.; Singha, S.; Jun, Y.W.; Reo, Y.J.; Kim, H.R.; Ryu, H.G.; Bhunia, S.; Ahn, K.H. Far-red/near-infrared emitting, two-photon absorbing, and bio-stable amino-Si-pyronin dyes. Chem. Sci. 2019, 10, 9028-9037. [CrossRef]

11. Lee, C.; Lee, S.; Kim, G.-U.; Lee, W.; Kim, B.J. Recent Advances, Design Guidelines, and Prospects of All-Polymer Solar Cells. Chem. Rev. 2019, 119, 8028-8086. [CrossRef] [PubMed]

12. Tamilavan, V.; Liu, Y.; Lee, J.; Shin, I.; Jung, Y.K.; Lee, B.R.; Jeong, J.H.; Park, S.H. Efficient Polymeric Donor for Both Visible and Near-Infrared-Absorbing Organic Solar Cells. ACS Appl. Energy Mater. 2019, 2, 4284-4291. [CrossRef]

13. Ding, F.; Zhan, Y.; Lu, X.; Sun, Y. Recent advances in near-infrared II fluorophores for multifunctional biomedical imaging. Chem. Sci. 2018, 9, 4370-4380. [CrossRef] 
14. Liu, T.-M.; Conde, J.; Lipinski, T.; Bednarkiewicz, A.; Huang, C.-C. Revisiting the classification of NIR-absorbing/emitting nanomaterials for in vivo bioapplications. Npg Asia Mater. 2016, 8, e295. [CrossRef]

15. Guo, Z.; Park, S.; Yoon, J.; Shin, I. Recent progress in the development of near-infrared fluorescent probes for bioimaging applications. Chem. Soc. Rev. 2014, 43, 16-29. [CrossRef] [PubMed]

16. Swamy, P.C.A.; Sivaraman, G.; Priyanka, R.N.; Raja, S.O.; Ponnuvel, K.; Shanmugpriya, J.; Gulyani, A. Near Infrared (NIR) absorbing dyes as promising photosensitizer for photodynamic therapy. Coord. Chem. Rev. 2020, 411, 213233. [CrossRef]

17. Fabian, J.; Nakazumi, H.; Matsuoka, M. Near infrared absorbing dyes. Chem. Rev. 1992, 92, 1197-1226. [CrossRef]

18. Freidus, L.G.; Pradeep, P.; Kumar, P.; Choonara, Y.E.; Pillay, V. Alternative fluorophores designed for advanced molecular imaging. Drug Discov. Today 2018, 23, 115-133. [CrossRef]

19. Luo, S.; Zhang, E.; Su, Y.; Cheng, T.; Shi, C. A review of NIR dyes in cancer targeting and imaging. Biomaterials 2011, 32, 7127-7138. [CrossRef]

20. Wu, D.; Chen, L.; Lee, W.; Ko, G.; Yin, J.; Yoon, J. Recent progress in the development of organic dye based near-infrared probes for metal ions. Coord. Chem. Rev. 2018, 354, 74-97. [CrossRef]

21. Mishra, A.; Behera, R.K.; Behera, P.K.; Mishra, B.K.; Behera, G.P. Cyanines during the 1990s: A Review. Chem. Rev. 2000, 100, 1973-2011. [CrossRef] [PubMed]

22. Shindy, H.A. Fundamentals in the chemistry of cyanine dyes: A review. Dye. Pigment. 2017, 145, 505-513. [CrossRef]

23. Ruppel, M.; Lungerich, D.; Sturm, S.; Lippert, R.; Hampel, F.; Jux, N. A Comprehensive Study on Tetraryltetrabenzoporhyrins. Chem. Eur. J. 2020, 26, 3287-3296. [CrossRef] [PubMed]

24. Li, X.; Zheng, B.-D.; Peng, X.-H.; Li, S.-Z.; Ying, J.-W.; Zhao, Y.; Huang, J.-D.; Yoon, J. Phtalocyanines as medicinal photosensitizers: Developments in the last five years. Coord. Chem. Rev. 2019, 379, 147-160. [CrossRef]

25. Demchenko, A.P. Photobleaching of organic fluorophores: Quantitative characterization, mechanisms, protection. Methods Appl. Fluoresc. 2020, 8, 022001. [CrossRef]

26. Bañuelos, J. BODIPY Dye, the Most Versatile Fluorophore Ever? Chem. Rec. 2016, 16, 335-348. [CrossRef]

27. Poddar, M.; Misra, R. Recent advances of BODIPY based derivatives for optoelectronic applications. Coord. Chem. Rev. 2020, 421, 213462. [CrossRef]

28. Boens, N.; Verbelen, B.; Ortiz, M.J.; Jiao, L.; Dehaen, W. Synthesis of BODIPY dyes through postfunctionalization of the boron dipyrromethene core. Coord. Chem. Rev. 2019, 399, 213024. [CrossRef]

29. Lu, H.; Mack, J.; Yang, Y.; Shen, Z. Structural modifications strategies for the rational design of red/NIR region BODIPYs. Chem. Soc. Rev. 2014, 43, 4778-4823. [CrossRef]

30. Martin, A.; Long, C.; Forster, R.J.; Keyes, T.E. Near IR emitting BODIPY fluorophores with mega-stokes shift. Chem. Commun. 2012, 48, 5617-5619. [CrossRef]

31. Ni, Y.; Kannadorai, R.K.; Yu, S.W.-K.; Chang, Y.-T.; Wu, J. Push-pull type meso-ester substituted BODIPY near-infrared dyes as contrast agents for photoacoustic imaging. Org. Biomol. Chem. 2017, 21, 4531-4535. [CrossRef]

32. Umezawa, K.; Matsui, A.; Nakamura, Y.; Citterio, D.; Suzuki, K. Bright, Color-Tunable Fluorescent Dyes in the Vis/NIR Region: Establishment of New "Tailor-Made" Multicolor Fluorophores Based on Borondipyrromethene. Chem. Eur. J. 2009, 15, 1096-1106. [CrossRef] [PubMed]

33. Li, Y.; Qiao, Z.; Li, T.; Zeika, O.; Leo, K. Highly Efficient Deep-Red- to Near-Infrared-Absorbing and Emissive Benzo/Naphthol[b]furan-Fused Boron Dipyrromethene (BODIPY). ChemPhotoChem 2018, 2, 1017-1021. [CrossRef]

34. Gómez-Durán, C.F.A.; Esnal, I.; Valois-Escamilla, I.; Urías-Benavides, A.; Bañuelos, J.; López-Arbeloa, I.; García-Moreno, I.; Peña-Cabrera, E. Near-IR BODIPY Dyes á la Carte-Programmed Orthogonal Functionalization of Rationally Designed Building Blocks. Chem. Eur. J. 2016, 22, 1048-1061. [CrossRef] [PubMed]

35. Verbelen, B.; Boodts, S.; Hofkens, J.; Boens, N.; Dehaen, W. Radical C-H Arylation of the BODIPY Core with Aryldiazonium Salts: Synthesis of Highly Fluorescent Red-Shifted Dyes. Angew. Chem. Int. Ed. 2015, 54, 4612-4616. [CrossRef] [PubMed] 
36. Bröring, M.; Krüger, R.; Link, S.; Kleeberg, C.; Köhler, S.; Xie, X.; Ventura, B.; Flamigni, L. Bis $\left(\mathrm{BF}_{2}\right)-2,2$ '-Bidipyrrins (BisBODIPYs): Highly Fluorescent BODIPY dimers with Large Stokes Shifts. Chem. Eur. J. 2008, 14, 2976-2983. [CrossRef] [PubMed]

37. Patalag, L.J.; Ho, L.P.; Jones, P.G.; Werz, D.B. Ethylene-bridged Oligo-BODIPYs: Access to Intramolecular J-Aggregates and Superfluorophores. J. Am. Chem. Soc. 2017, 139, 15104-15113. [CrossRef]

38. Nakamura, M.; Tahara, H.; Takahashi, K.; Nagata, T.; Uoyama, H.; Kuzuhara, D.; Mori, S.; Okujima, T.; Yamada, H.; Uno, H. $\pi$-Fused bis-BODIPY as candidate for NIR dyes. Org. Biomol. Chem. 2012, 10, 6840-6849. [CrossRef]

39. Yu, C.; Jiao, L.; Li, T.; Wu, Q.; Miao, W.; Wang, J.; Wei, Y.; Mu, X.; Hao, E. Fusion and planarization of bisBODIPY: A new family of photostable near infrared dyes. Chem. Commun. 2015, 51, 16852-16855. [CrossRef]

40. Ni, Y.; Lee, S.; Son, M.; Aratani, N.; Ishida, M.; Samanta, A.; Yamada, H.; Chang, Y.-T.; Furuta, H.; Kim, D.; et al. A Diradical Approach towards BODIPY-Based Dyes with Intense Near-Infrared Absorption around $\lambda$ $=1100 \mathrm{~nm}$. Angew. Chem. Int. Ed. 2016, 55, 2815-2819. [CrossRef]

41. Zhao, W.; Carreira, E.M. Conformationally Restrcited Aza-BODIPY: Highly Fluorescent, Stable Near-Infrared Absorbing Dyes. Chem. Eur. J. 2006, 12, 7254-7263. [CrossRef] [PubMed]

42. Ge, Y.; O'Shea, D.F. Azadipyrromethenes: From traditional dye chemistry to leading edge applications. Chem. Soc. Rev. 2016, 45, 3846-3864. [CrossRef] [PubMed]

43. Cui, J.; Sheng, W.; Wu, Q.; Yu, C.; Hao, E.; Bobadova-Parvanova, P.; Storer, M.; Asiri, A.M.; Marwani, H.M.; Jiao, J. Synthesis, Structure, and Properties of Near-Infrared [b]Phenanthrene-Fused $\mathrm{BF}_{2}$ Azadipyrromethenes. Chem. Asian J. 2017, 12, 2486-2493. [CrossRef] [PubMed]

44. Karlsson, J.K.G.; Harriman, A. Origin of the Red-Shifted Optical Spectra recorded for Aza-BODIPY Dyes. J. Phys. Chem. A 2016, 120, 2537-2546. [CrossRef] [PubMed]

45. Kuehne, A.J.C.; Gather, M.C. Organic Lasers: Recent Developments of Materials, Device Geometries, and Fabrication Techniques. Chem. Rev. 2016, 116, 12823-12864. [CrossRef] [PubMed]

46. Jiang, Y.; Liu, Y.-Y.; Liu, X.; Lin, H.; Gao, K.; Lai, W.-Y.; Huang, W. Organic solid-state lasers: A materials view and future developments. Chem. Soc. Rev. 2020. [CrossRef]

47. Belmonte-Vázquez, J.L.; Avellanal-Zaballa, E.; Enríquez-Palacios, E.; Cerdán, L.; Esnal, I.; Bañuelos, J.; Villegas-Gómez, C.; López-Arbeloa, I.; Peña-Cabrera, E. Synthetic Approach to Readily Accessible Benzofuran-Fused Borondipyrromethenes as Red-Emitting Laser Dyes. J. Org. Chem. 2019, 84, 2523-2541. [CrossRef]

48. Prieto-Castañeda, A.; Avellanal-Zaballa, E.; Gartzia-Rivero, L.; Cerdán, L.; Agarrabeitia, A.R.; García-Moreno, I.; Bañuelos, J.; Ortiz, M.J. Tailoring the Molecular Skeleton of Aza-BODIPYs to Design Photostable Red-Light-Emitting Laser Dyes. ChemPhotoChem 2019, 3, 75-85. [CrossRef]

49. Lin, Z.; Kohn, A.W.; Voorhis, T.V. Toward Prediction of Nonradiative Decay Pathways in Organic Compounds II: Two Internal Conversion Channels in BODIPYs. J. Phys. Chem. C 2020, 124, 3925-3938. [CrossRef]

50. Mei, J.; Leung, N.L.C.; Kwok, R.T.K.; Lam, J.W.Y.; Tang, B.Z. Aggregation-Induced Emission: Together We Shine, United We Soar! Chem. Rev. 2015, 115, 11708-11940. [CrossRef]

51. Cerdán, L.; Martínez-Martínez, V.; García-Moreno, I.; Costela, A.; Pérez-Ojeda, M.E.; López-Arbeloa, I.; Wu, L.; Burgess, K. Naturally Asembled Excimers in Xanthenes as Singular and Highly Efficient Laser Dyes in Liquid and Solid Media. Adv. Opt. Mater. 2013, 1, 984-990. [CrossRef]

52. Durán-Sampedro, G.; Agarrabeitia, A.R.; Arbeloa López, T.; Bañuelos, J.; López-Arbeloa, I.; Chiara, J.L.; Ortiz, M.J. Increased laser action in commercial dyes form fluorination regardless of their skeleton. Laser Phys. Lett. 2014, 11, 115818. [CrossRef]

53. López-Arbeloa, F.; Bañuelos Prieto, J.; Martínez Martínez, V.; Arbeloa López, T.; López Arbeloa, I. Intramolecular Charge Transfer in Pyrromethene Laser Dyes: Photophysical behaviour of PM650. ChemPhysChem 2004, 5, 1762-1771. [CrossRef]

54. Englman, R.; Jortner, J. The energy gap law for non-radiative decay in large molecules. J. Lumin. 1970, 1, 134-142. [CrossRef]

55. Cerdán, L.; Enciso, E.; Martin, V.; Bañuelos, J.; López-Arbeloa, I.; Costela, A.; García-Moreno, I. FRET-assisted laser emission in colloidal suspensions of dye-doped latex nanoparticles. Nat. Photon. 2012, 6, 621-626. [CrossRef] 
56. Durán-Sampedro, G.; Agarrabeitia, A.R.; Cerdán, L.; Pérez-Ojeda, M.E.; Costela, A.; García-Moreno, I.; Esnal, I.; Bañuelos, J.; López-Arbeloa, I.; Ortiz, M.J. Carboxylates versus Fluorines: Boosting the Emission Properties of Commercial BODIPYs in Liquid and Solid Media. Adv. Funct. Mater. 2013, 23, 4195-4205.

57. Durán-Sampedro, G.; Esnal, I.; Agarrabeitia, A.R.; Bañuelos Prieto, J.; Cerdán, L.; García-Moreno, I.; Costela, A.; López-Arbeloa, I.; Ortiz, M.J. First Highly Efficient and Photostable $E$ and $C$ Derivatives of 4,4-Difluoro-4-bora-3a,4a-diaza-s-indacene (BODIPY) as Dye Lasers in the Liquid Phase, Thin Films, and Solid-State Rods. Chem. Eur. J. 2014, 20, 2646-2653. [CrossRef]

58. Bodio, W.; Goze, C. Investigation of B-F substitution on BODIPY and aza-BODIPY dyes: Development of B-O and B-C BODIPYs. Dye. Pigment. 2019, 160, 700-710. [CrossRef]

(C) 2020 by the authors. Licensee MDPI, Basel, Switzerland. This article is an open access article distributed under the terms and conditions of the Creative Commons Attribution (CC BY) license (http://creativecommons.org/licenses/by/4.0/). 